\title{
GROTHENDIECK RINGS FOR LIE SUPERALGEBRAS AND THE DUFLO-SERGANOVA FUNCTOR
}

\author{
CRYSTAL HOYT AND SHIFRA REIF
}

\begin{abstract}
We show that the Duflo-Serganova functor on the category of finite-dimensional modules over a finite-dimensional contragredient Lie superalgebra induces a ring homomorphism on a natural quotient of the Grothendieck ring, which is isomorphic to the ring of supercharacters. We realize this homomorphism as a certain evaluation of functions related to the supersymmetry property. We use this realization to describe the kernel and image of the homomorphism induced by the Duflo-Serganova functor.
\end{abstract}

\section{INTRODUCTION}

The Duflo-Serganova functor was originally introduced in [DS together with associated varieties of modules over Lie superalgebras. On the category of finite-dimensional modules, the Duflo-Serganova functor is a tensor functor which preserves the superdimension. This functor was used by Serganova in [S11] to prove the conjecture of Kac and Wakimoto that the superdimension of a finite-dimensional module is zero if and only if the atypicality of the module is maximal. The Duflo-Serganova functor was also used to give an additional proof for the superdimension formula of $G L(m \mid n)$-modules in [HW1], and has been applied to study Deligne categories in $\mathrm{CH}$, EHS, $\mathrm{H}$, HW2.

Given an odd element $x$ in a Lie superalgebra $\mathfrak{g}$ satisfying $[x, x]=0$, we have that $x^{2}=0$ in the universal enveloping algebra of $\mathfrak{g}$, and so for every $\mathfrak{g}$-module $M$, we can define the cohomology

$$
M_{x}:=\operatorname{Ker}_{M} x / x M .
$$

In fact, $M_{x}$ is a module for the Lie superalgebra

$$
\mathfrak{g}_{x}:=\operatorname{Ker} \operatorname{ad}_{x} / \operatorname{Im} \operatorname{ad}_{x},
$$

which is a Lie superalgebra of smaller rank than $\mathfrak{g}$. For example, if $\mathfrak{g}=\mathfrak{g l}(m \mid n)$ and $x$ is a root vector, then $\mathfrak{g}_{x}=\mathfrak{g l}(m-1 \mid n-1)$. Duflo and Serganova defined the functor $D S_{x}: M \mapsto M_{x}$ from the category of $\mathfrak{g}$-modules to the category of $\mathfrak{g}_{x}$-modules [DS], which we refer to as the Duflo-Serganova functor.

One of the difficulties that arises in using the Duflo-Serganova functor is that it is not exact. It is therefore surprising that it induces a ring homomorphism $d s_{x}$ on a natural quotient of the Grothendieck ring of the category of finite-dimensional $\mathfrak{g}$-modules. This quotient is defined by identifying the equivalence class of a module $[M]$ with $-[\Pi(M)]$, where $\Pi$ is the shift of parity functor. We refer to this quotient as the supercharacter ring of $\mathfrak{g}$ and show that the homomorphism $d s_{x}$ is indeed well defined.

Sergeev and Veselov described the supercharacter ring as a ring of functions admitting a certain supersymmetry condition in [SV]. In this paper, we realize the homomorphism $d s_{x}$ in terms of evaluation of functions related to the supersymmetry condition. For example, the supercharacter ring of the Lie supergroup $G L(m \mid n)$ corresponding to the Lie superalgebra $\mathfrak{g l}(m \mid n)$ is isomorphic to the ring of doubly symmetric Laurent polynomials in $x_{1}, \ldots, x_{m}, y_{1}, \ldots, y_{n}$ for which the evaluation

The first author was partially supported by BSF Grant 2012227. The second author was partially supported by ORT Braude College's Research Authority. 
$x_{1}=y_{1}=t$ is independent of $t$. If $x$ is a root vector for the root $\varepsilon_{i}-\delta_{j}$ of $\mathfrak{g l}(m \mid n)$, then the homomorphism $d s_{x}$ is given by the evaluation $x_{i}=y_{j}=t$, which is independent of the variable $t$ after evaluation, by the supersymmetry property.

We use this realization to describe the kernel of the homomorphism $d s_{x}$ when $x$ is a root vector. In particular, we show that if $\mathfrak{g}$ is a Lie superalgebra of Type I, the supercharacters of Kac modules form a basis for the kernel. When $\mathfrak{g}$ is a Lie superalgebra of Type II, there are no Kac modules; however, we show that the kernel has a basis consisting of expressions similar to the supercharacters of Kac modules. These are the same expressions that were used by Gruson and Serganova to define Kazhdan-Lusztig polynomials for the orthosymplectic Lie superalgebras GS].

We also describe the image of $d s_{x}$. In particular, for $\mathfrak{g}=\mathfrak{s l}(m \mid n), m \neq n$, and $\mathfrak{o s p}(m \mid 2 n)$, we show that the image is the supercharacter ring of $G_{x}$, where $G_{x}$ is the Lie supergroup corresponding to the Lie superalgebra $\mathfrak{g}_{x}$. Moreover, we prove that the homomorphism induced by the Duflo-Serganova functor from the category of finite-dimensional $G$-modules to the category of finite-dimensional $G_{x}$-modules is surjective. For the exceptional Lie superalgebras, we explicitly describe the image using a set of generators.

Acknowledgment. The authors are thankful to Maria Gorelik, Rachel Karpman, Ivan Penkov and Vera Serganova for fruitful conversations.

\section{PRELiminaRies}

2.1. Lie superalgebras. Lie superalgebras are a natural generalization of Lie algebras which first appeared in mathematical physics. In this paper, we study the finite-dimensional contragredient Lie superalgebras $\mathfrak{g}=\mathfrak{g}_{\overline{0}} \oplus \mathfrak{g}_{\overline{1}}$ with indecomposable Cartan matrix. These are the Lie superalgebras $\mathfrak{s l}(m \mid n) \quad m \neq n, \mathfrak{g l}(n \mid n), \mathfrak{o s p}(m \mid 2 n), D(2,1, \alpha), F(4)$ or $\mathrm{G}(3)$. We also consider the case when $\mathfrak{g}=\mathfrak{g l}(m \mid n)$ is the general linear Lie superalgebra. These Lie superalgebras resemble reductive Lie algebras in their structure theory; in particular, they are defined by a Cartan matrix and they possess an even supersymmetric invariant bilinear form $(\cdot, \cdot)$ which has kernel equal to the center of $\mathfrak{g}$.

Fix a Cartan subalgebra $\mathfrak{h} \subset \mathfrak{g}_{\overline{0}} \subset \mathfrak{g}$, and consider the corresponding root space decomposition

$$
\mathfrak{g}=\mathfrak{h} \oplus \bigoplus_{\alpha \in \Delta} \mathfrak{g}_{\alpha}
$$

Then the set of roots $\Delta \subset \mathfrak{h}^{*}$ splits $\Delta=\Delta_{\overline{0}} \sqcup \Delta_{\overline{1}}$ into even roots $\Delta_{\overline{0}}$ and odd roots $\Delta_{\overline{1}}$. A choice of positive roots $\Delta^{+}=\Delta_{\overline{0}}^{+} \sqcup \Delta_{\overline{1}}^{+}$determines a triangular decomposition of $\mathfrak{g}$ given by $\mathfrak{g}=\mathfrak{n}^{+} \oplus \mathfrak{h} \oplus \mathfrak{n}^{-}$, where $\mathfrak{n}^{ \pm}=\oplus_{\alpha \in \Delta^{ \pm}} \mathfrak{g}_{\alpha}$. Let $\rho_{\overline{0}}=\frac{1}{2} \sum_{\alpha \in \Delta_{\overline{0}}^{+}} \alpha, \rho_{\overline{1}}=\frac{1}{2} \sum_{\alpha \in \Delta_{\overline{1}}^{+}} \alpha$ and $\rho=\rho_{\overline{0}}-\rho_{\overline{1}}$. The Weyl group $W$ of $\mathfrak{g}$ is by definition the Weyl group of $\mathfrak{g}_{\overline{0}}$. The sign map $\operatorname{sgn}: W \rightarrow\{ \pm 1\}$ is defined by $w \mapsto(-1)^{l(w)}$, where $l(w)$ denotes the length of $w$ as a product of simple reflections with respect to a set of simple roots for $\mathfrak{g}_{\overline{0}}$.

The space $\mathfrak{h}^{*}$ inherits an even supersymmetric bilinear form $(\cdot, \cdot)$. A root $\beta \in \Delta_{\overline{1}}$ is called isotropic if $(\beta, \beta)=0$. Two roots $\alpha, \beta \in \Delta$ are called orthogonal if $(\alpha, \beta)=0$. The maximal number of linearly independent mutually orthogonal isotropic roots is called the defect of $\mathfrak{g}$. We denote by $\Delta_{\text {iso }}:=\left\{\beta \in \Delta_{\overline{1}} \mid(\beta, \beta)=0\right\}$ the set of all isotropic roots, by $\Delta_{\text {iso }}^{+}=\Delta_{\text {iso }} \cap \Delta^{+}$the set of positive isotropic roots and we let $\rho_{i s o}:=\frac{1}{2} \sum_{\alpha \in \Delta_{i s o}^{+}} \alpha$. We define

$$
\mathcal{S}_{\mathfrak{g}}=\left\{B \subset \Delta_{\text {iso }} \mid B=\left\{\beta_{1}, \ldots, \beta_{k} \mid\left(\beta_{i}, \beta_{j}\right)=0, \beta_{i} \neq \pm \beta_{j}\right\}\right\}
$$

to be the set of subsets of linearly independent mutually orthogonal isotropic roots.

The space $\mathfrak{h}^{*}$ has a natural basis $\varepsilon_{1}, \ldots, \varepsilon_{m}, \delta_{1}, \ldots, \delta_{n}$, which for $\mathfrak{g l}(m \mid n)$ and $\mathfrak{o s p}(m \mid 2 n)$ satisfies $\left(\varepsilon_{i}, \varepsilon_{j}\right)=\delta_{i j}=-\left(\delta_{i}, \delta_{j}\right)$ and $\left(\varepsilon_{i}, \delta_{j}\right)=0$. The roots of $\mathfrak{g}$ have a nice presentation in this basis (see [CW, $\mathrm{Mu}$ for more details). Let $Q_{\mathfrak{g}}=\operatorname{span}_{\mathbb{Z}} \Delta$ be the root lattice of $\mathfrak{g}$, and let $Q_{\mathfrak{g}}^{+}=\operatorname{span}_{\mathbb{Z}} \Delta^{+}$. 
The parity function $p: \Delta \rightarrow \mathbb{Z}_{2}$ extends uniquely to a linear function $p: Q_{\mathfrak{g}} \rightarrow \mathbb{Z}_{2}$. The root lattice $Q_{\mathfrak{g}}$ is contained in the integral weight lattice $P_{\overline{0}}$ for $\mathfrak{g}_{\overline{0}}$, where

$$
P_{\overline{0}}=\left\{\lambda \in \mathfrak{h}^{*} \mid \frac{2(\lambda, \alpha)}{(\alpha, \alpha)} \in \mathbb{Z} \forall \alpha \in \Delta_{\overline{0}}\right\} .
$$

The set of dominant integral weights

$$
P_{\overline{0}}^{+}=\left\{\lambda \in P_{\overline{0}} \mid \frac{2(\lambda, \alpha)}{(\alpha, \alpha)} \geq 0 \forall \alpha \in \Delta_{\overline{0}}\right\}
$$

is the set of highest weights of finite-dimensional simple $\mathfrak{g}_{0}$-modules.

The category of finite-dimensional modules $\mathcal{F}_{\mathfrak{g}}$ over a Lie superalgebra $\mathfrak{g}$ is not semisimple, that is, there exist indecomposable modules which are not irreducible. For example, a Lie superalgebra $\mathfrak{g}$ of Type I has a decomposition $\mathfrak{g}=\mathfrak{g}_{-1} \oplus \mathfrak{g}_{\overline{0}} \oplus \mathfrak{g}_{+1}$, so one can define the Kac module of highest weight $\lambda \in P_{\overline{0}}$ as

$$
K(\lambda)=\operatorname{Ind}_{\mathfrak{g}_{\overline{0}} \oplus \mathfrak{g}_{+1}}^{\mathfrak{g}} \mathrm{L}_{\overline{0}}(\lambda),
$$

where $L_{\overline{0}}(\lambda)$ is the finite-dimensional simple $\mathfrak{g}_{\overline{0}}$-module of highest weight $\lambda$ and $\mathfrak{g}_{+1}$ acts trivially on $L_{\overline{0}}(\lambda)$. Then $K(\lambda)$ is a finite-dimensional, indecomposable $\mathfrak{g}$-module with a unique simple quotient $L(\lambda)$, where $\lambda$ is the highest weight with respect to the distinguished choice of simple roots, and $K(\lambda)$ is simple (i.e. $K(\lambda)=L(\lambda))$ if and only if $\lambda$ is a typical weight: $(\lambda+\rho, \beta) \neq 0$ for all $\beta \in \Delta_{\text {iso }}$ (see, for example, CW, Chapter 2] for more details).

If $G_{\overline{0}}$ is a simply-connected connected Lie group corresponding to the Lie algebra $\mathfrak{g}_{\overline{0}}$ [S14], and $\mathcal{F}_{G}$ is the full subcategory of $\mathcal{F}_{\mathfrak{g}}$ consisting of all finite-dimensional $G_{\overline{0}}$-integrable modules, then $\mathcal{F}_{G}$ is equivalent to the category of finite-dimensional modules over the corresponding algebraic supergroup $G$ [S14].

2.2. Supercharacter rings of Lie superalgebras. The character theory of Lie superalgebras is a rich area of research which has led to interesting applications in number theory (see [KW1, KW2]). For a finite-dimensional $\mathfrak{g}$-module $M$, with weight decomposition $M=\bigoplus_{\mu \in \mathfrak{h}^{*}} M^{\mu}$ and weight spaces $M^{\mu}=M_{\overline{0}}^{\mu} \oplus M_{\overline{1}}^{\mu}$, the supercharacter of $M$ is defined to be

$$
\operatorname{sch} M=\sum_{\mu \in \mathfrak{h}^{*}}\left(\operatorname{dim} M_{\overline{0}}^{\mu}-\operatorname{dim} M_{\overline{1}}^{\mu}\right) e^{\mu},
$$

while the character of $M$ is given by $\operatorname{ch} M=\sum\left(\operatorname{dim} M_{\overline{0}}^{\mu}+\operatorname{dim} M_{\overline{1}}^{\mu}\right) e^{\mu}$. A finite-dimensional simple $\mathfrak{g}$-module is determined by its supercharacter, as well as by its character [SV, Prop. 4.2].

The supercharacter ring $\mathcal{J}_{\mathfrak{g}}$ of a Lie superalgebra $\mathfrak{g}$ is defined to be the image of the map

$$
\operatorname{sch}: \mathcal{F}_{\mathfrak{g}} \rightarrow \mathbb{Z}\left[P_{\overline{0}}\right]^{W},
$$

where $\mathbb{Z}\left[P_{\overline{0}}\right]:=\mathbb{Z}\left\{e^{\mu} \mid \mu \in P_{\overline{0}}\right\}$. For an element $f \in \mathcal{J}_{\mathfrak{g}}$, with $f=\sum_{\mu \in P_{\overline{0}}} c_{\mu} e^{\mu}$, we call the set Supp $f=\left\{\mu \in P_{\overline{0}} \mid c_{\mu} \neq 0\right\}$ the support of $f$.

For a fixed choice of positive roots $\Delta^{+}=\Delta_{\overline{0}}^{+} \sqcup \Delta_{\overline{1}}^{+}$, we denote the super Weyl denominator by $R=\frac{R_{\overline{0}}}{R_{\overline{1}}}$ where $R_{\overline{0}}=\prod_{\alpha \in \Delta_{\overline{0}}^{+}}\left(1-e^{-\alpha}\right)$ and $R_{\overline{1}}=\prod_{\alpha \in \Delta_{\overline{1}}^{+}}\left(1-e^{-\alpha}\right)$. Note that the supercharacter of the Kac module equals

$$
\operatorname{sch} K(\lambda)=e^{-\rho} R^{-1} \cdot \operatorname{ch} L_{\overline{0}}(\lambda),
$$

where $\Delta^{+}=\Delta_{\overline{0}}^{+} \sqcup \Delta_{\overline{1}}^{+}$is the distinguished choice of simple roots.

The Grothendieck group of the category $\mathcal{F}_{\mathfrak{g}}$ is defined by taking the free abelian group generated by the elements $[M]$ which represent each isomorphism class of finite-dimensional $\mathfrak{g}$-modules, and modding out by the relations $\left[M_{1}\right]-\left[M_{2}\right]+\left[M_{3}\right]$ for all exact sequences $0 \rightarrow M_{1} \rightarrow M_{2} \rightarrow M_{3} \rightarrow 0$. Since $\mathcal{F}_{\mathfrak{g}}$ is closed under tensor products, the Grothendieck group inherits a natural ring structure. 
The Grothendieck ring of $\mathcal{F}_{\mathfrak{g}}$ has a natural quotient described as follows. Let $\Pi$ denote the parity reversing functor from $\mathcal{F}_{\mathfrak{g}}$ to itself, and let $\mathcal{K}_{\mathfrak{g}}$ denote the quotient of the Grothendieck ring of $\mathcal{F}_{\mathfrak{g}}$ by the ideal $\langle[\Pi(M)]+[M]| M$ is a $\mathfrak{g}$-module $\rangle$. The map sch : $\mathcal{K}_{\mathfrak{g}} \rightarrow \mathbb{Z}\left[P_{\overline{0}}\right]^{W_{\overline{0}}}$ given on generators by $[M] \mapsto \operatorname{sch} M$ is injective [SV, Proposition 4.4], and its image is the supercharacter ring $\mathcal{J}_{\mathfrak{g}}$ of $\mathfrak{g}$.

Remark 1. In this paper, we identify the rings $\mathcal{K}_{\mathfrak{g}}$ and $\mathcal{J}_{\mathfrak{g}}$ under this isomorphism, and use the notation $\mathcal{J}_{\mathfrak{g}}$ to denote this ring. Given a module $M \in \mathcal{F}_{\mathfrak{g}}$, we write $[M]$ for its image in $\mathcal{J}_{\mathfrak{g}}$.

Sergeev and Veselov gave an explicit description of supercharacter rings for basic classical Lie superalgebras in $[\mathrm{SV}]$ as follows. The supercharacter ring of $\mathfrak{g}$ is isomorphic to the the space of supersymmetric exponential functions

$$
\mathcal{J}_{\mathfrak{g}}=\left\{f \in \mathbb{Z}\left[P_{\overline{0}}\right]^{W} \mid D_{\beta} f \text { is in the ideal generated by }\left(e^{\beta}-1\right) \text { for any } \beta \in \Delta_{\text {iso }}\right\}
$$

where $D_{\beta}\left(e^{\lambda}\right)=(\lambda, \beta) e^{\beta}$. Sergeev and Veselov also described the supercharacter $\operatorname{ring} \mathcal{J}_{G} \subset \mathcal{J}_{\mathfrak{g}}$ for the Lie supergroup $G$ corresponding to the Lie superalgebra $\mathfrak{g}$ as a ring of Laurent polynomials subject to some additional conditions [SV, Section 7]. Recall the basis $\varepsilon_{1}, \ldots, \varepsilon_{m}, \delta_{1}, \ldots, \delta_{n}$ of $\mathfrak{h}^{*}$, and define: $x_{i}:=e^{\varepsilon_{i}}, y_{j}:=e^{\delta_{j}}, u_{i}=x_{i}+x_{i}^{-1}, v_{j}=y_{j}+y_{j}^{-1}$.

$G L(m \mid n)$ : The supercharacter ring of $G L(m \mid n)$ is

$$
\mathcal{J}_{G}=\left\{f \in \mathbb{Z}\left[x_{1}^{ \pm 1}, \ldots, x_{m}^{ \pm 1}, y_{1}^{ \pm 1}, \ldots, y_{n}^{ \pm 1}\right]^{S_{m} \times S_{n}} \mid y_{j} \frac{\partial f}{\partial y_{j}}+x_{i} \frac{\partial f}{\partial x_{i}} \in\left\langle y_{j}-x_{i}\right\rangle\right\} .
$$

$S L(m \mid n), \quad m \neq n$ : The supercharacter ring of $S L(m \mid n)$ for $m \neq n$ is the quotient of (2.3) by the ideal $\left\langle x_{1} \ldots x_{m}-y_{1} \ldots y_{n}\right\rangle$.

$B(m \mid n): \quad$ The supercharacter ring of $O S P(2 m+1 \mid 2 n)$ is

$$
\mathcal{J}_{G}=\left\{f \in \mathbb{Z}\left[u_{1}, \ldots, u_{m}, v_{1}, \ldots, v_{n}\right]^{S_{m} \times S_{n}} \mid u_{i} \frac{\partial f}{\partial u_{i}}+v_{j} \frac{\partial f}{\partial v_{j}} \in\left\langle u_{i}-v_{j}\right\rangle\right\} .
$$

$C(n+1)$ : The supercharacter ring of $O S P(2 \mid 2 n)$ is

$$
\mathcal{J}_{G}=\left\{f \in \mathbb{Z}\left[u_{1}, v_{1}, \ldots, v_{n}\right]^{S_{m}} \mid u_{1} \frac{\partial f}{\partial u_{1}}+v_{j} \frac{\partial f}{\partial v_{j}} \in\left\langle u_{1}-v_{j}\right\rangle\right\} .
$$

$D(m \mid n), \quad m \geq 2:$ The supercharacter ring of $O S P(2 m \mid 2 n)$ for $m \geq 2$ is

$$
\mathcal{J}_{G}=\left\{f \in \mathbb{Z}\left[u_{1}, \ldots, u_{m}, v_{1}, \ldots, v_{n}\right]^{S_{m} \times S_{n}} \mid u_{i} \frac{\partial f}{\partial u_{i}}+v_{j} \frac{\partial f}{\partial v_{j}} \in\left\langle u_{i}-v_{j}\right\rangle\right\} .
$$

Remark 2. Note that $f \in \mathcal{J}_{G L(m \mid n)}$ if and only if it is supersymmetric in $x_{1}, \ldots, x_{m}, y_{1}, \ldots y_{n}$, that is, if it is invariant under permutation of $x_{1}, \ldots, x_{m}$ and of $y_{1}, \ldots, y_{n}$, and if the substitution $x_{1}=y_{1}=t$ made in $f$ is independent of $t$ (see for example [Mu, Section 12]).

2.3. The Duflo-Serganova functor. The idea behind the Duflo-Serganova functor is simple and natural. For any odd element $x \in \mathfrak{g}_{\overline{1}}$ of a finite-dimensional contragredient Lie superalgebra $\mathfrak{g}$ which satisfies $[x, x]=0$, we have that $x^{2}=0$ in the universal enveloping algebra of $\mathfrak{g}$, and so for any finite-dimensional $\mathfrak{g}$-module $M$ we can define the cohomology

$$
M_{x}:=\operatorname{Ker}_{M} x / x M \text {. }
$$

Then $M_{x}$ is in fact a module over the Lie superalgebra

$$
\mathfrak{g}_{x}:=\mathfrak{g}^{x} /[x, \mathfrak{g}],
$$


where $\mathfrak{g}^{x}=\{a \in \mathfrak{g} \mid[x, a]=0\}$ is the centralizer of $x$ in $\mathfrak{g}$ [DS, Lemma 6.2]. The Duflo-Serganova functor $D S_{x}: \mathcal{F}_{\mathfrak{g}} \rightarrow \mathcal{F}_{\mathfrak{g}_{x}}$ is defined from the category of finite-dimensional $\mathfrak{g}$-modules to the category of finite-dimensional $\mathfrak{g}_{x}$-modules by sending $M \mapsto M_{x}$.

The Duflo-Serganova functor is a cohomology functor and hence is a symmetric monoidal tensor functor, that is, for $\mathfrak{g}$-modules $M, N$ one has a natural isomorphism $M_{x} \otimes N_{x} \rightarrow(M \otimes N)_{x}$ [S11]. Moreover, the Duflo-Serganova functor commutes with direct sums, however it is not exact.

Let $X_{\mathfrak{g}}=\left\{x \in \mathfrak{g}_{\overline{1}}:[x, x]=0\right\}$, and let $\mathcal{S}_{\mathfrak{g}}$ be the set of subsets of mutually orthogonal isotropic roots (see (2.1) ). Then the $G_{\overline{0}}$-orbits of $X_{\mathfrak{g}}$ are in one-to-one correspondence with the $W$-orbits of $\mathcal{S}_{\mathfrak{g}}$ via the correspondence

$$
B=\left\{\beta_{1}, \ldots, \beta_{k}\right\} \mapsto x=x_{\beta_{1}}+\cdots+x_{\beta_{k}} \in X_{\mathfrak{g}},
$$

where each $x_{\beta_{i}} \in \mathfrak{g}_{\beta_{\mathrm{i}}}$ is chosen to be nonzero [DS, Theorem 4.2].

The Lie superalgebra $\mathfrak{g}_{x}$ can be naturally embedded into $\mathfrak{g}^{x} \subset \mathfrak{g}$, in such a way that $\mathfrak{h}_{x}=\mathfrak{h} \cap \mathfrak{g}_{x}$ is a Cartan subalgebra of $\mathfrak{g}_{x}$ and the root spaces of $\mathfrak{g}_{x}$ are root spaces of $\mathfrak{g}$ [DS, Lemma 6.3]. More explicitly, Duflo and Serganova proved the following.

If $B=\left\{\beta_{1}, \ldots, \beta_{k}\right\} \in \mathcal{S}$ and $x=x_{\beta_{1}}+\cdots+x_{\beta_{k}}$ for some nonzero $x_{\beta_{i}} \in \mathfrak{g}_{\beta_{\mathfrak{i}}}$, then $\mathfrak{g}^{x} \subset \mathfrak{g}$ can be decomposed into a semidirect sum $\mathfrak{g}^{x}=[x, \mathfrak{g}] \Subset \mathfrak{g}_{x}$, where $\mathfrak{g}_{x}=\mathfrak{h}_{x} \oplus\left(\oplus_{\alpha \in \Delta_{x}} \mathfrak{g}_{\alpha}\right)$, the subspace $\mathfrak{h}_{x}=\mathfrak{h} \cap \mathfrak{g}_{x}$ is a Cartan subalgebra of $\mathfrak{g}_{x}$ and

$$
\Delta_{x}=\{\alpha \in \Delta \mid(\alpha, \beta)=0 \forall \beta \in B \text { and } \pm \alpha \notin B\}
$$

is the root system of $\mathfrak{g}_{x}$.

For each finite-dimensional contragredient Lie superalgebra $\mathfrak{g}$ with irreducible Cartan matrix, we can explicitly describe the isomorphism type of $\mathfrak{g}_{x}$. If $\mathcal{B}=\left\{\beta_{1}, \ldots, \beta_{k}\right\} \in \mathcal{S}$ and $x=x_{\beta_{1}}+\cdots+x_{\beta_{k}}$ for some nonzero $x_{\beta_{i}} \in \mathfrak{g}_{\beta_{\mathrm{i}}}$, then by [DS, Remark 6.4] we have the following. In particular, the defect of $\mathfrak{g}_{x}$ equals the defect of $\mathfrak{g}$ minus $k$.

\begin{tabular}{|c||c|c|c|c|c|c|}
\hline $\mathfrak{g}$ & $\mathfrak{g l}(m \mid n)$ & $\mathfrak{s l}(m \mid n), m \neq n$ & $\mathfrak{o s p}(m \mid 2 n)$ & $D(2,1, \alpha)$ & $F_{4}$ & $G_{3}$ \\
\hline & & & & & & \\
$\mathfrak{g}_{x}$ & $\mathfrak{g l}(m-k \mid n-k)$ & $\mathfrak{s l}(m-k \mid n-k)$ & $\mathfrak{o} \mathfrak{s p}(m-2 k \mid 2 n-2 k)$ & $\mathbb{C}$ & $\mathfrak{s l}(3)$ & $\mathfrak{s l}(2)$ \\
\hline
\end{tabular}

Remark 3. Note that when $\mathfrak{g}_{x}$ is simple, the embedding $\mathfrak{g}^{x} \subset \mathfrak{g}$ is determined by the condition that the root spaces of $\mathfrak{g}_{x}$ are mapped into the respective root spaces of $\mathfrak{g}$, since in this case $\mathfrak{h}_{x} \subset\left[\mathfrak{n}_{x}^{+}, \mathfrak{n}_{x}^{-}\right]$. For $\mathfrak{g}=\mathfrak{g l}(m, n)$, we take the matrix embedding of $\mathfrak{g}_{x}=\mathfrak{g l}(m-k \mid n-k)$ into $\mathfrak{g l}(m \mid n)$ which has $2 k$ zero rows and $2 k$ zero columns at the locations $r_{i}, n+s_{i}$, for $i=1, \ldots, k$, when $B=\left\{\beta_{i}=\varepsilon_{r_{i}}-\delta_{s_{i}}\right\}_{i=1, \ldots, k}$ is the set of maximal isotropic roots defining $x$.

\section{The Duflo--Serganova Functor And the SUPercharaCter Ring}

In this section, we prove that the Duflo-Serganova functor $D S_{x}: \mathcal{F}_{\mathfrak{g}} \rightarrow \mathcal{F}_{\mathfrak{g}_{x}}$ induces a ring homomorphism $d s_{x}: \mathcal{J}_{\mathfrak{g}} \rightarrow \mathcal{J}_{\mathfrak{g}_{x}}$, and we realize it as a certain evaluation of the functions $f \in \mathcal{J}_{\mathfrak{g}}$ related to the supersymmetry property defining $\mathcal{J}_{\mathfrak{g}}$.

3.1. The ring homomorphism induced by the Duflo-Serganova functor. Let $\mathfrak{g}$ be a finitedimensional contragredient Lie superalgebra with indecomposable Cartan matrix or let $\mathfrak{g}=\mathfrak{g l}(m, n)$, and fix a Cartan subalgebra $\mathfrak{h}$ of $\mathfrak{g}$. Let $B=\left\{\beta_{1}, \ldots, \beta_{k}\right\} \in \mathcal{S}_{\mathfrak{g}}, x \in X_{\mathfrak{g}}$ and $x=x_{\beta_{1}}+\cdots+x_{\beta_{k}}$ for nonzero $x_{\beta_{i}} \in \mathfrak{g}_{\beta_{\mathfrak{i}}}$. Fix an embedding $\mathfrak{g}_{x} \subset \mathfrak{g}^{x} \subset \mathfrak{g}$ with Cartan subalgebra $\mathfrak{h}_{x}=\mathfrak{h} \cap \mathfrak{g}_{x}$ (see Section 2.3 .

Lemma 4. For $\mathfrak{g}$-modules $M$ and $N$ we have

(1) $\operatorname{sch} M_{x}(h)=\operatorname{sch} M(h)$ for all $h \in \mathfrak{h}_{x}$;

(2) if $\operatorname{sch} M=\operatorname{sch} N$, then $\operatorname{sch} M_{x}=\operatorname{sch} N_{x}$. 
Proof. We have an exact sequence $0 \rightarrow \operatorname{ker}_{M} x \rightarrow M \rightarrow x M \rightarrow 0$ of $\mathfrak{h}^{x}$-invariant spaces. Thus, $M / \operatorname{ker}_{M} x \cong \Pi(x M)$ as $\mathfrak{h}^{x}$-modules, where $\Pi$ switches the parity of a superspace, and $\operatorname{so~} \operatorname{sch}\left(M / \operatorname{ker}_{M} x\right)(h)=\operatorname{sch} \Pi(x M)(h)$ for all $h \in \mathfrak{h}^{x}$. Hence, for all $h \in \mathfrak{h}_{x} \subset \mathfrak{h}^{x}$ we have that

$\operatorname{sch} M(h)=\operatorname{sch} \operatorname{ker} x(h)+\operatorname{sch} \Pi(x M)(h)=\operatorname{sch} \operatorname{ker} x(h)-\operatorname{sch} M(h)=\operatorname{sch}\left(\operatorname{ker}_{M} x / x M\right)(h)$

$$
=\operatorname{sch}\left(M_{x}\right)(h) \text {. }
$$

Remark 5. The following example shows that Lemma 4 does not hold if we replace supercharacter by character. It also shows that the Duflo-Serganova functor is not exact.

Example 6. Let $\mathfrak{g}=\mathfrak{g l}(2 \mid 1)$ with the standard choice of simple roots $\left\{\alpha=\varepsilon_{1}-\varepsilon_{2}, \beta=\varepsilon_{2}-\delta_{1}\right\}$. Let $K(0)$ be the Kac module with highest weight zero, and denote the highest weight vector by $v_{0}$. Then $K(0)=\operatorname{span}\left\{v_{0}, f_{\beta} v_{0}, f_{\alpha+\beta} v_{0}, f_{\beta} f_{\alpha+\beta} v_{0}\right\}$, where $f_{\beta} \in \mathfrak{g}_{-\beta}$ and $f_{\alpha+\beta} \in \mathfrak{g}_{-\alpha-\beta}$ are nonzero. The maximal submodule of $K(0)$ is $\bar{K}(0):=\operatorname{span}\left\{f_{\beta} v_{0}, f_{\alpha+\beta} v_{0}, f_{\beta} f_{\alpha+\beta} v_{0}\right\}$, and the simple quotient of $K(0)$ is isomorphic to the trivial $\mathfrak{g}$-module $L(0)$. Clearly, the $\mathfrak{g}$-modules $K(0)$ and $L(0) \oplus \bar{K}(0)$ have the same character and supercharacter.

Let us show that for $x=f_{\beta}$, the $\mathfrak{g}_{x}$-modules $K(0)_{x}$ and $(L(0) \oplus \bar{K}(0))_{x}$ have the same supercharacter but not the same character. In this case, $\mathfrak{g}_{x}=\mathfrak{g l}(1 \mid 0)$. By a direct computation using (2.4) and the basis given above, one can check that $K(0)_{x}=\{0\}, L(0)_{x} \cong \mathbb{C}_{1 \mid 0}$ and $\bar{K}(0)_{x} \cong \mathbb{C}_{0 \mid 1}$, where $\mathbb{C}_{1 \mid 0}$ (resp. $\left.\mathbb{C}_{0 \mid 1}\right)$ is the even (resp. odd) trivial $\mathfrak{g}_{x}$-module. Thus, $\operatorname{ch} K(0)_{x}=\operatorname{sch} K(0)_{x}=0$ and $\operatorname{sch}(L(0) \oplus \bar{K}(0))_{x}=0$, while $\operatorname{ch}(L(0) \oplus \bar{K}(0))_{x}=2$.

Definition 7. We define $d s_{x}: \mathcal{J}_{\mathfrak{g}} \rightarrow \mathcal{J}_{\mathfrak{g}_{x}}$ on the generators $[M] \in \mathcal{J}_{\mathfrak{g}}$, where $M \in \mathcal{F}_{\mathfrak{g}}$, by

$$
d s_{x}([M])=\left[D S_{x}(M)\right]
$$

and we extend linearly to $\mathcal{J}_{\mathfrak{g}}$.

It is not difficult to show that $d s_{x}$ is a well-defined linear map using Lemma 4. The fact that $d s_{x}$ is a ring homomorphism then follows from the fact that $D S_{x}$ is a tensor functor. Hence, we have

Proposition 8. Let $\mathfrak{g}$ be a finite-dimensional contragredient Lie superalgebra, and let $x \in \mathfrak{g}_{1}$ nonzero such that $[x, x]=0$. The functor $D S_{x}: \mathcal{F}_{\mathfrak{g}} \rightarrow \mathcal{F}_{\mathfrak{g}_{x}}$ induces a ring homomorphism on the corresponding supercharacter rings $d s_{x}: \mathcal{J}_{\mathfrak{g}} \rightarrow \mathcal{J}_{\mathfrak{g}_{x}}$.

Remark 9. The proofs in Section 3.1 also work for modules in the BGG Category $\mathcal{O}$, and so the Duflo-Serganova functor induces a group homomorphism on the quotient of the Grothendieck group by the parity. However, it is not a ring homomorphism since Category $\mathcal{O}$ is not closed under tensor products.

3.2. Realization of the ring homomorphism. Given $f \in \mathcal{J}_{\mathfrak{g}}$ we can realize $f: \mathfrak{h} \rightarrow \mathbb{C}$ as a supersymmetric function in the variables $x_{1}, \ldots, x_{m}, y_{1}, \ldots, y_{n}$ with $x_{i}=e^{\varepsilon_{i}}$ and $y_{j}=e^{\delta_{j}}$, using the supercharacter ring description of Sergeev and Veselov (see Equation 2.2). (Note that for $F(4)$ we take $x_{i}=e^{\frac{1}{2} \varepsilon_{i}}$ and $y_{j}=e^{\frac{1}{2} \delta_{j}}$.)

Theorem 10. Suppose $d s_{x}: \mathcal{J}_{\mathfrak{g}} \rightarrow \mathcal{J}_{\mathfrak{g}_{x}}$ is defined by $x=x_{\beta_{1}}+\cdots+x_{\beta_{k}}$ for nonzero $x_{\beta_{i}} \in \mathfrak{g}_{\beta_{\mathfrak{i}}}$, where $B=\left\{\beta_{1}, \ldots, \beta_{k}\right\} \in \mathcal{S}_{\mathfrak{g}}$.

(1) Then for any $f \in \mathcal{J}_{\mathfrak{g}}$,

$$
d s_{x}(f)=\left.f\right|_{\mathfrak{h}_{x}} .
$$

(2) If $B=\left\{\varepsilon_{1}-\delta_{1}\right\}$, then $d s_{x}(f)$ is given by substituting $x_{1}=y_{1}$ into $f$, that is,

$$
d s_{x} f=\left.f\right|_{x_{1}=y_{1}} .
$$


If $\mathfrak{g}=F(4)($ resp. $D(2,1, \alpha))$ and $B=\left\{\frac{1}{2}\left(\varepsilon_{1}+\varepsilon_{2}+\varepsilon_{3}-\delta_{1}\right)\right\}$ (resp. $\left.B=\left\{\varepsilon_{1}-\varepsilon_{2}-\varepsilon_{3}\right\}\right)$, then $d s_{x} f$ is given by substituting $y_{1}=x_{1} x_{2} x_{3}$ (respectively, $x_{1}=x_{2} x_{3}$ ) into $f$.

(3) If $B=\left\{\beta_{i}=a_{i} \varepsilon_{r_{i}}-b_{i} \delta_{s_{i}}\right\}_{i=1, \ldots, k}$ for some $a_{i}, b_{i} \in\{ \pm 1\}$, then $d s_{x} f$ is given by substituting $x_{r_{i}}^{a_{i}}=y_{s_{i}}^{b_{i}}$ into $f$, that is,

(4) For any $f \in \mathcal{J}_{\mathfrak{g}}$,

$$
d s_{x} f=\left.f\right|_{x_{r_{i}}^{a_{i}}=y_{s}^{b_{i}} ; i=1, \ldots, k} .
$$

$$
d s_{x}(f)=\left.f\right|_{\beta_{1}=\cdots=\beta_{k}=0} .
$$

Proof. It suffices to prove (1) for a spanning set of $\mathcal{J}_{\mathfrak{g}}$. Suppose $[M] \in \mathcal{K}_{\mathfrak{g}}$ corresponds to a module $M \in \mathcal{F}_{\mathfrak{g}}$. By Lemma 团, we have

$$
d s_{x}([M])=\left[D S_{x}(M)\right]=\operatorname{sch} M_{x}=\left.(\operatorname{sch} M)\right|_{\mathfrak{h}_{x}}=\left.[M]\right|_{\mathfrak{h}_{x}} \in \mathcal{J}_{\mathfrak{g}_{x}} .
$$

Hence, $d s_{x}(f)=\left.f\right|_{\mathfrak{h}_{x}}$ for any $f \in \mathcal{J}_{\mathfrak{g}}$.

To prove (2), fix $f \in \mathcal{J}_{\mathfrak{g}}$, and suppose that $x \in \mathfrak{g}_{\beta}$. If $\beta=\varepsilon_{1}-\delta_{1}$, then the evaluation $f_{x_{1}=y_{1}=t}$ is well defined and independent of $t$ due to the supersymmetry property of $f \in \mathcal{J}_{\mathfrak{g}}$. Thus,

$$
\left.f\right|_{x_{1}=y_{1}=t}:=f\left(t, x_{2}, \ldots, x_{m} \mid t, y_{2}, \ldots, y_{n}\right)
$$

is equal to the restriction of $f$ to the hyperplane $x_{1}-y_{1}=0$. Since $\mathfrak{h}_{x} \subset \mathfrak{h}^{x}=\{h \in \mathfrak{h} \mid \beta(h)=0\}$ belongs to the hyperplane $x_{1}-y_{1}=0$, we have proven that $d s_{x} f=\left.f\right|_{\mathfrak{h}_{x}}=\left.f\right|_{x_{1}=y_{1}}$. The cases $\beta=\varepsilon_{1}+\varepsilon_{2}+\varepsilon_{3}-\delta_{1}$ and $\beta=\varepsilon_{1}-\varepsilon_{2}-\varepsilon_{3}$ are similar.

Now (3) can be proven using arguments similar to that of (2) and the fact that

$$
\mathfrak{h}^{x}=\{h \in \mathfrak{h} \mid \beta(h)=0 \text { for all } \beta \in B\} .
$$

Finally, (4) follows from (3) since if $\beta_{i}=a_{i} \varepsilon_{r_{i}}-b_{i} \delta_{r_{i}}$, then $\beta_{i}=0$ if and only if $x_{r_{i}}^{a_{i}} y_{r_{i}}^{-b_{i}}=e^{\beta_{i}}=1$ if and only if $x_{r_{i}}^{a_{i}}=y_{r_{i}}^{b_{i}}$.

Corollary 11. If $x=x_{\beta_{1}}+\ldots+x_{\beta_{k}}$ where $x_{\beta_{i}} \in \mathfrak{g}_{\beta_{i}}$ and $B=\left\{\beta_{1}, \ldots, \beta_{k}\right\} \in \mathcal{S}$, then for all $f \in \mathcal{J}_{\mathfrak{g}}$

$$
d s_{x}(f)=d s_{x_{\beta_{1}}} \circ \ldots \circ d s_{x_{\beta_{k}}}(f) .
$$

\section{THE KeRNEL OF THE RING HOMOMORPHISM}

In this section, we give a $\mathbb{Z}$-basis for the kernel of $d s_{x}$ when $x \in \mathfrak{g}_{\beta}$ is a root vector of an isotropic $\operatorname{root} \beta$ for the Lie superalgebra $\mathfrak{g}$. Our basis is given by elements of the following form.

Definition 12. For each $\lambda \in P_{\overline{0}}$, we define

$$
k(\lambda):=R^{-1} \cdot \sum_{w \in W}(-1)^{l(w)+p(w(\rho)-\rho)} e^{w(\lambda+\rho)-\rho} .
$$

Here $p(w(\rho)-\rho)$ denotes the parity of $w(\rho)-\rho$, which is well-defined since $w(\rho)-\rho \in Q$. Note that the element $w(\rho)-\rho$ may be odd, e.g. in osp $(1 \mid 2)$.

For each $\lambda \in P_{\overline{0}}^{+}$, the expression $k(\lambda)$ is in $\mathbb{Z}\left[P_{\overline{0}}\right]^{W}$ since it is the product of the $W$-invariant polynomial $e^{\rho_{\overline{1}}} R_{\overline{1}}$ and the character of a finite-dimensional $\mathfrak{g}_{\overline{0}}$-module given by the Weyl character formula. Moreover, since the evaluation $\left.k(\lambda)\right|_{\beta=0}$ equals zero for any $\beta \in \Delta_{\text {iso }}$ we have that $k(\lambda) \in \mathcal{J}_{\mathfrak{g}}$. It is clear that $k(\lambda)$ is in the kernel of $d s_{x}$ for any $x \in \mathfrak{g}_{\beta}$, since $d s_{x}\left(R_{\overline{1}}\right)=0$.

For Lie superalgebras of Type I with the distinguished choice of simple roots, $k(\lambda)$ is the supercharacter of a Kac module when $\lambda \in P_{\overline{0}}^{+}$, whereas in Type II, $k(\lambda)$ is a virtual supercharacter. Similar virtual characters were used by Gruson and Serganova in [GS] to study the character formula of simple modules over orthosymplectic Lie superalgebras.

We need the following definition to prove the main result in this section for Lie superalgebras of Type II. 
Definition 13. Given a finite-dimensional Lie superalgebra $\mathfrak{g}$ with root system $\Delta$, we define a Lie algebra $\widetilde{\mathfrak{g}}$ as follows. We let $\widetilde{\Delta}$ be the root system with positive even roots given by

$$
\widetilde{\Delta}^{+}:=\left\{\alpha \in \Delta_{\overline{0}}^{+} \mid \frac{\alpha}{2} \notin \Delta_{\overline{1}}\right\} \cup\left\{\alpha \in \Delta_{\overline{1}}^{+} \mid \alpha \notin \Delta_{i s o}\right\}
$$

and we let $\tilde{\mathfrak{g}}$ be the semisimple Lie algebra with root system $\widetilde{\Delta}$. If $\Delta_{\overline{1}}=\Delta_{i s o}$, then $\tilde{\mathfrak{g}}=\mathfrak{g}_{\overline{0}}$. If $\mathfrak{g}=B(m \mid n), G(3)$ then $\widetilde{\mathfrak{g}} \cong B_{m} \times B_{n}, G_{2} \times A_{1}$, respectively. We set $\widetilde{\rho}:=\frac{1}{2} \sum_{\alpha \in \widetilde{\Delta}^{+}} \alpha$. Note that $\rho=\widetilde{\rho}-\rho_{\text {iso }}$, since $\beta \in \Delta_{\text {iso }}$ if and only if $\beta \in \Delta_{\overline{1}}$ but $2 \beta \notin \Delta_{\overline{0}}$. Let $P_{\widetilde{\mathfrak{g}}}^{+}$denote the set of dominant integral weights of $\widetilde{\mathfrak{g}}$. Then $P_{\overline{0}}^{+} \subset P_{\mathfrak{\mathfrak { g }}}^{+}$and the Weyl group of $\widetilde{\mathfrak{g}}$ is isomorphic to the Weyl group of $\mathfrak{g}_{\overline{0}}$. We extend the definition of $k(\lambda)$ to $\lambda \in P_{\mathfrak{\mathfrak { g }}}$ by letting

$$
k(\lambda):=R^{-1} \cdot \sum_{w \in W}(-1)^{l(w)+p(w(\lambda+\rho)-\rho)} e^{w(\lambda+\rho)-\rho} .
$$

We have the following lemma.

Lemma 14. The set $\left\{k(\mu) \mid \mu \in P_{\mathfrak{g}}^{+}+\rho_{\text {iso }}\right\}$ is linearly independent.

Proof. To prove linear independence we consider a completion of $\mathbb{Z}\left[P_{\mathfrak{g}}\right]$, where we allow expansions in the domain $\left|e^{-\alpha}\right|<1$ for $\alpha \in \widetilde{\Delta}^{+}$. Note that in this completion, $R^{-1}=\sum_{\nu \in-Q_{\tilde{\mathfrak{g}}}^{+}} b_{\nu} e^{\nu}$ for some $b_{\nu} \in \mathbb{Z}$. For each $\mu \in P_{\widetilde{\mathfrak{g}}}^{+}+\rho_{\text {iso }}$, we will show that $\mu+\rho$ is a strictly dominant element of $P_{\mathfrak{g}}$, that is, $w(\mu+\rho)<\mu+\rho$ for $w \in W, w \neq 1$. Indeed, if $\mu \in P_{\tilde{\mathfrak{g}}}^{+}+\rho_{\text {iso }}$, then $\mu+\rho=\lambda+\widetilde{\rho}$ for some $\lambda \in P_{\tilde{\mathfrak{g}}}^{+}$. Since $\lambda+\widetilde{\rho}$ is strictly dominant with respect to $\tilde{\mathfrak{g}}$, it is also strictly dominant with respect to $\mathfrak{g}_{\overline{0}}$ and the claim follows. Thus,

$$
k(\mu)=e^{\mu}+\sum_{\nu \in \mu-Q_{\mathfrak{\mathfrak { g }}}^{+}} a_{\nu} e^{\nu}
$$

and linear independence follows.

Remark 15. Note that if one takes the distinguished choice of simple roots for $\mathfrak{g l}(m, n)$, then $P^{+}=P^{+}+\rho_{\text {iso }}$, since in this case $\left(\rho_{i s o}, \alpha\right)=0$ for every even root $\alpha$.

The following lemma is used in the proof of the main theorem of this section.

Lemma 16. For each $\mu \in P_{\widetilde{\mathfrak{g}}}^{+}$, we have $k\left(\mu+\rho_{\text {iso }}\right)=e^{\rho_{\text {iso }}} \prod_{\alpha \in \Delta_{\text {iso }}^{+}}\left(1-e^{-\alpha}\right) \cdot \operatorname{ch} L_{\tilde{\mathfrak{g}}}(\mu)$.

Proof. For any element $g \in \mathbb{Z}\left[P_{\mathfrak{\mathfrak { g }}}\right]$ with $\operatorname{Supp} g \subset \mu+Q_{\widetilde{\mathfrak{g}}}$, we write $g=\sum_{\lambda \in Q_{\mathfrak{\mathfrak { g }}}} c_{\mu+\lambda} e^{\mu+\lambda}$, and we define $\bar{g}=\sum_{\lambda \in Q_{\mathfrak{g}}}(-1)^{p(\lambda)} c_{\mu+\lambda} e^{\mu+\lambda}$, where $p: Q_{\widetilde{\mathfrak{g}}} \rightarrow \mathbb{Z}_{2}$ is the parity function. Clearly, this operation is an involution. So we have that

$\overline{e^{\rho_{i s o}} \prod_{\alpha \in \Delta_{i s o}^{+}}\left(1-e^{-\alpha}\right) \cdot \operatorname{ch} L_{\tilde{\mathfrak{g}}}(\mu)}=$ 


$$
\begin{aligned}
& =(-1)^{p\left(\rho_{i s o}\right)} e^{\rho_{i s o}} \prod_{\alpha \in \Delta_{i s o}^{+}}\left(1+e^{-\alpha}\right) \cdot \operatorname{sch} L_{\tilde{g}}(\mu) \\
& =(-1)^{p\left(\rho_{i s o}\right)} e^{\rho_{i s o}} \prod_{\alpha \in \Delta_{i s o}^{+}}\left(1+e^{-\alpha}\right) \frac{\sum_{w \in W}(-1)^{l(w)+p(w(\mu+\widetilde{\rho})-\widetilde{\rho})} e^{w(\mu+\widetilde{\rho})-\widetilde{\rho}}}{\prod_{\alpha \in \widetilde{\Delta}_{\overline{0}}^{+}}\left(1-e^{-\alpha}\right)} \\
& =\prod_{\alpha \in \Delta_{\overline{1}}^{+}}\left(1+e^{-\alpha}\right) \cdot \frac{\sum_{w \in W}(-1)^{l(w)+p\left(w\left(\mu+\rho_{i s o}+\rho\right)-\rho\right)} e^{w\left(\left(\mu+\rho_{i s o}\right)+\widetilde{\rho}-\rho_{i s o}\right)-\widetilde{\rho}+\rho_{i s o}}}{\prod_{\alpha \in \widetilde{\Delta}_{\overline{0}}^{+}}\left(1-e^{-\alpha}\right) \prod_{\alpha \in \Delta_{\overline{1}}^{+} \backslash \Delta_{i s o}^{+}}\left(1+e^{-\alpha}\right)} \\
& =\prod_{\alpha \in \Delta_{\overline{1}}^{+}}\left(1+e^{-\alpha}\right) \frac{\sum_{w \in W}(-1)^{l(w)+p\left(w\left(\left(\mu+\rho_{i s o}\right)+\rho\right)-\rho\right)} e^{w\left(\left(\mu+\rho_{i s o}\right)+\rho\right)-\rho}}{\prod_{\alpha \in \Delta_{\overline{0}}^{+}}\left(1-e^{-\alpha}\right)} \\
& =\frac{k\left(\mu+\rho_{i s o}\right)}{k(\mu)}
\end{aligned}
$$

and hence the claim follows.

We have the following theorem.

Theorem 17. If $\beta$ is an odd isotropic root and $x \in \mathfrak{g}_{\beta}$, then the set

$$
\left\{k(\lambda) \mid \lambda \in P_{\overline{0}}^{+}+\rho_{\text {iso }}\right\}
$$

is a $\mathbb{Z}$-basis for the kernel of $d s_{x}: \mathcal{J}_{\mathfrak{g}} \rightarrow \mathcal{J}_{\mathfrak{g}_{x}}$.

Proof. Linear independence of the set (4.1) follows from Lemma 14 since $P_{\overline{0}}^{+} \subset P_{\tilde{\mathfrak{g}}}^{+}$. So it only remains to show that the set (4.1) spans the kernel of $d s_{x}: \mathcal{J}_{\mathfrak{g}} \rightarrow \mathcal{J}_{\mathfrak{g}_{x}}$.

Let $f \in \mathcal{J}_{\mathfrak{g}}$ such that $d s_{x}(f)=0$. According to Theorem 10, this means that the restriction of $f$ to the hyperplane $\beta=0$ is zero, or equivalently, substituting $e^{-\beta}=1$ yields zero. Hence, $f$ is divisible by $\left(1-e^{-\beta}\right)$. Since $f$ is $W$-invariant and $W \beta=\Delta_{i s o}$, it follows that $f$ is divisible by $e^{\rho_{\text {iso }}} \prod_{\alpha \in \Delta_{\text {iso }}^{+}}\left(1-e^{-\alpha}\right)$.

Write

$$
f=e^{\rho_{\text {iso }}} \prod_{\alpha \in \Delta_{i s o}^{+}}\left(1-e^{-\alpha}\right) \cdot g .
$$

Then $g$ is a $W$-invariant element of $\mathbb{Z}\left[P_{\overline{0}}\right]$, since both $f$ and $e^{\rho_{\text {iso }}} \prod_{\alpha \in \Delta_{\text {iso }}^{+}}\left(1-e^{-\alpha}\right)$ are $W$-invariant.

Case 1: First suppose that $\mathfrak{g}$ does not have non-isotropic roots, then $\Delta_{\text {iso }}^{+}=\Delta_{\overline{1}}^{+}$and $\rho_{\text {iso }}=\rho_{\overline{1}}$. By the theory of symmetric functions,

$$
g=\sum_{\mu \in P_{\overline{0}}^{+}}^{\text {finite }} a_{\mu} \operatorname{ch} L_{\mathfrak{g}_{\overline{0}}}(\mu),
$$

for some $a_{\mu} \in \mathbb{Z}$, where $P_{\overline{0}}^{+}$is the set of highest weights of finite-dimensional $\mathfrak{g}_{\overline{0}}$-modules (see for example $\underline{\mathrm{Ma}}$ ]. 
By the Weyl character formula for semisimple Lie algebras, we have that

$$
\begin{aligned}
f & =e^{\rho_{\overline{1}}} R_{\overline{1}} \cdot g \\
& =e^{\rho_{\overline{1}}} R_{\overline{1}} \sum_{\mu \in P_{\overline{0}}^{+}} a_{\mu} \operatorname{ch} L_{\mathfrak{g}_{\overline{0}}}(\mu) \\
& =e^{\rho_{\overline{1}}} R_{\overline{1}} \sum_{\mu \in P_{\overline{0}}^{+}} a_{\mu} \frac{\sum_{w \in W}(-1)^{l(w)} e^{w\left(\mu+\rho_{0}\right)}}{e^{\rho_{\overline{0}}} R_{\overline{0}}} \\
& =e^{\rho_{\overline{1}}} R_{\overline{1}} \sum_{\lambda \in P_{\overline{0}}^{+}+\rho_{\overline{1}}} b_{\lambda} \frac{\sum_{w \in W}(-1)^{l(w)} e^{w\left(\lambda+\rho_{0}-\rho_{1}\right)}}{e^{\rho_{\overline{0}} R_{\overline{0}}}} \\
& =\sum_{\lambda \in P_{\overline{0}}^{+}+\rho_{\overline{1}}} b_{\lambda} k(\lambda),
\end{aligned}
$$

where $b_{\lambda}:=a_{\lambda-\rho_{\overline{1}}}$. For each $w \in W$, the parity of $w(\rho)$ equals the parity of $\rho$, since $\rho \in P_{\overline{0}}$. Hence, the last equality follows.

Case 2: Suppose that $\mathfrak{g}$ has non-isotropic roots. Since $P_{\overline{0}} \subset P_{\mathfrak{\mathfrak { g }}}$, by the theory of characters of Lie algebras

$$
g=\sum_{\mu \in P_{\mathfrak{\mathfrak { g }}}^{+}}^{\text {finite }} a_{\mu} \operatorname{ch} L_{\mathfrak{g}}(\mu)
$$

for some $a_{\mu} \in \mathbb{Z}$. By Lemma [16, we have that

$$
\begin{aligned}
f & =e^{\rho_{i s o}} \prod_{\alpha \in \Delta_{\text {iso }}^{+}}\left(1-e^{-\alpha}\right) \cdot g \\
& =e^{\rho_{i s o}} \prod_{\alpha \in \Delta_{\text {iso }}^{+}}\left(1-e^{-\alpha}\right) \sum_{\mu \in P_{\tilde{\mathfrak{g}}}^{+}} a_{\mu} \operatorname{ch} L_{\tilde{\mathfrak{g}}}(\mu) \\
& =\sum_{\mu \in P_{\mathfrak{\mathfrak { g }}}^{+}} a_{\mu} \cdot e^{\rho_{i s o}} \prod_{\alpha \in \Delta_{\text {iso }}^{+}}\left(1-e^{-\alpha}\right) \cdot \operatorname{ch} L_{\tilde{\mathfrak{g}}}(\mu) \\
& =\sum_{\mu \in P_{\tilde{\mathfrak{g}}}^{+}} a_{\mu} \cdot k\left(\mu+\rho_{\text {iso }}\right) \\
& =\sum_{\lambda \in P_{\tilde{\mathfrak{g}}}^{+}+\rho_{\text {iso }}} b_{\lambda} k(\lambda)
\end{aligned}
$$

where $b_{\lambda}:=a_{\lambda-\rho_{\text {iso }}}$. We are left to show that $b_{\lambda}=0$ for $\lambda \notin P_{\overline{0}}^{+}+\rho_{\text {iso }}$. Since $\operatorname{Supp} f \subset P_{\overline{0}}$, Supp $k(\lambda) \subset P_{\overline{0}}$, the elements $k(\lambda)$ for $\mu \in P_{\widetilde{\mathfrak{g}}}^{+}+\rho_{\text {iso }}$ are linearly independent and the sum in (4.2) is finite, we conclude that

$$
f=\sum_{\lambda \in P_{\overline{0}}^{+}+\rho_{\text {iso }}} b_{\lambda} k(\lambda) .
$$

Corollary 18. Let $G$ be one of the Lie supergroups $S L(m \mid n), m \neq n, G L(m \mid n)$ or $S O S P(m \mid 2 n)$, and let $\mathfrak{g}$ be the corresponding Lie superalgebra. Let $\beta$ be an odd isotropic root, $x \in \mathfrak{g}_{\beta}$ and let $D S_{x}: \mathcal{F}_{G} \rightarrow \mathcal{F}_{G_{x}}$ be the Duflo-Serganova functor from the category $\mathcal{F}_{G}$ of finite-dimensional 
$G$-modules to the category $\mathcal{F}_{G_{x}}$ of finite-dimensional $G_{x}$-modules, where $G_{x}$ denotes the Lie supergroup corresponding to the Lie superalgebra $\mathfrak{g}_{x}$. Then the kernel of the induced ring homomorphism $d s_{x}: \mathcal{J}_{G} \rightarrow \mathcal{J}_{G_{x}}$ has a $\mathbb{Z}$-basis

$$
\left\{k(\lambda) \mid \lambda \in P_{G}^{+}+\rho_{\text {iso }}\right\},
$$

where $P_{G}^{+}$is the set of highest weights for finite-dimensional $G$-modules.

Proof. Let $P_{G} \subset P_{\overline{0}}$ be the sublattice of integral weights of finite-dimensional $G_{\overline{0}}$-modules. Then for $G=G L(m \mid n)$ or $\operatorname{SOSP}(m \mid 2 n)$

$$
P_{G}=\left\{\sum_{i=1}^{m} \lambda_{i} \varepsilon_{i}+\sum_{j=1}^{n} \mu_{j} \delta_{j} \mid \lambda_{i}, \mu_{j} \in \mathbb{Z}\right\}
$$

and the supercharacter ring for the category of finite-dimensional $G$-modules $\mathcal{F}_{G}$ is

$$
\mathcal{J}_{G}=\left\{f \in \mathbb{Z}\left[x_{1}^{ \pm 1}, \ldots, x_{m}^{ \pm 1}, y_{1}^{ \pm 1}, \ldots, y_{n}^{ \pm 1}\right]^{W} \mid y_{j} \frac{\partial f}{\partial y_{j}}+x_{i} \frac{\partial f}{\partial x_{i}} \in\left\langle y_{j}-x_{i}\right\rangle\right\}
$$

as shown in [SV, Section 7] (note that this ring is denoted by $J(\mathfrak{g})_{0}$ in [SV]). If $G=S L(m \mid n)$, $m \neq n$, then

$$
P_{G}=\left\{\sum_{i=1}^{m} \lambda_{i} \varepsilon_{i}+\sum_{j=1}^{n} \mu_{j} \delta_{j} \mid \lambda_{i}, \mu_{j} \in \mathbb{Z}, \sum_{i=1}^{m} \lambda_{i}-\sum_{j=1}^{n} \mu_{j}=0\right\},
$$

and the supercharacter ring for the category of finite-dimensional $G$-modules $\mathcal{F}_{G}$ is

$$
\mathcal{J}_{G}=\left\{f \in \mathbb{Z}\left[x_{1}^{ \pm 1}, \ldots, x_{m}^{ \pm 1}, y_{1}^{ \pm 1}, \ldots, y_{n}^{ \pm 1}\right]^{W} /\left\langle x_{1} \cdots x_{m}-y_{1} \cdots y_{n}\right\rangle \mid y_{j} \frac{\partial f}{\partial y_{j}}+x_{i} \frac{\partial f}{\partial x_{i}} \in\left\langle y_{j}-x_{i}\right\rangle\right\}
$$

as shown in $\left[\mathrm{SV}\right.$, Section 7]. Since in both cases $\mathcal{J}_{G}=\mathcal{J}_{\mathfrak{g}} \cap \mathbb{Z}\left[P_{G}\right]$, the kernel of the homomorphism $d s_{x}: \mathcal{J}_{G} \rightarrow \mathcal{J}_{G_{x}}$ equals $\operatorname{Ker}_{G} d s_{x}=\operatorname{Ker}_{\mathfrak{g}} d s_{x} \cap \mathbb{Z}\left[P_{G}\right]$, where $\operatorname{Ker}_{\mathfrak{g}} d s_{x}$ is the kernel of the corresponding homomorphism $d s_{x}: \mathcal{J}_{\mathfrak{g}} \rightarrow \mathcal{J}_{\mathfrak{g}_{x}}$. It follows from the linear independence of the elements $k(\lambda)$ and the fact that $\lambda \in P_{G}$ if and only if $\operatorname{Supp} k(\lambda) \in P_{G}$, that $\operatorname{Ker}_{G} d s_{x}=\operatorname{span}\left\{k(\lambda) \mid \lambda \in P_{G}+\rho_{\text {iso }}\right\}$. Since $P_{G}^{+}=P_{\mathfrak{g}}^{+} \cap P_{G}$, the claim follows.

Remark 19. On the level of categories, it was shown in [BKN that a module $M$ over a Type I finite-dimensional contragredient Lie superalgebra has a filtration of Kac modules (resp. dual Kac modules) if and only if $D S_{x}(M)=0$ for all $x \in X_{\mathfrak{g}}^{-}$(resp. $x \in X_{\mathfrak{g}}^{+}$), where $X_{\mathfrak{g}}^{ \pm}=X_{\mathfrak{g}} \cap \mathfrak{n}^{ \pm}$and $\mathfrak{g}=\mathfrak{n}^{-} \oplus \mathfrak{h} \oplus \mathfrak{n}^{+}$is the triangular decomposition with respect to the distinguished choice of simple roots.

\section{The image of the Ring homomorphism}

5.1. Image of $d s_{x}$ for classical Lie superalgebras. Let $\mathfrak{g}$ be one of the Lie superalgebras: $\mathfrak{s l}(m \mid n), m \neq n, \mathfrak{g l}(m \mid n)$ and $\mathfrak{o s p}(m \mid 2 n)$. In this section, we describe the image of $d s_{x}$ for every $x \in X_{\mathfrak{g}}$. We use the realization of $d s_{x}$ given in Theorem 10, and the explicit description of the supercharacter rings given by Sergeev and Veselov in [SV, Section 7].

Theorem 20. Let $G$ be one of the Lie supergroups $S L(m \mid n), m \neq n, G L(m \mid n)$ or OSP $(m, 2 n)$ and let $\mathfrak{g}$ be the corresponding Lie superalgebra. For any $x \in X_{\mathfrak{g}}$, the Duflo-Serganova functor $D S_{x}: \mathcal{F}_{G} \rightarrow \mathcal{F}_{G_{x}}$ from the category $\mathcal{F}_{G}$ of finite-dimensional $G$-modules to the category $\mathcal{F}_{G_{x}}$ of finite-dimensional $G_{x}$-modules induces a surjective ring homomorphism on the corresponding supercharacter rings $d s_{x}: \mathcal{J}_{G} \rightarrow \mathcal{J}_{G_{x}}$. 
Proof. We will use Corollary 11 to reduce to the case that $x \in \mathfrak{g}_{\beta}$ for some isotropic root $\beta$. Using the realization of $d s_{x}$ given in Theorem 10 we will show that $d s_{x}$ transfers a certain set of generators of the supercharacter ring $\mathcal{K}_{G}$ to a set of generators of the supercharacter ring $\mathcal{J}_{G_{x}}$. We use the same set of generators of $\mathcal{J}_{G}$ that Sergeev and Veselov used to give explicit descriptions of supercharacter rings over basic Lie superalgebras and their corresponding Lie supergroups [SV, Section 7].

$G L(m, n)$ : The supercharacter ring of $G L(m, n)$ is generated by $\frac{x_{1} \cdots x_{m}}{y_{1} \cdots y_{n}}, \frac{y_{1} \cdots y_{n}}{x_{1} \cdots x_{m}}, h_{k}\left(x_{1}, \ldots, x_{m}, y_{1}, \ldots, y_{n}\right)$, $h_{k}\left(x_{1}^{-1}, \ldots, x_{m}^{-1}, y_{1}^{-1}, \ldots, y_{n}^{-1}\right), k \in \mathbb{Z}_{>0}$, where

$$
\chi_{G}(t)=\frac{\prod_{i=1}^{m}\left(1-x_{i} t\right)}{\prod_{j=1}^{n}\left(1-y_{j} t\right)}=\sum_{k=0}^{\infty} h_{k}\left(x_{1}, \ldots, x_{m}, y_{1}, \ldots, y_{n}\right) t^{k} .
$$

$S L(m, n), m \neq n$ : The supercharacter ring of $S L(m, n), m \neq n$, is generated by $h_{k}\left(x_{1}, \ldots, x_{m}, y_{1}, \ldots, y_{n}\right)$, $h_{k}\left(x_{1}^{-1}, \ldots, x_{m}^{-1}, y_{1}^{-1}, \ldots, y_{n}^{-1}\right), k \in \mathbb{Z}_{>0}$, where $h_{k}$ is given by (5.1).

$O S P(2 m+1,2 n)$ : The supercharacter ring of $O S P(2 m+1,2 n)$ is generated by $h_{k}\left(x_{1}, \ldots, x_{m}, y_{1}, \ldots, y_{n}\right)$, $k \in \mathbb{Z}_{>0}$ where

$$
\chi_{G}(t)=\frac{\prod_{j=1}^{n}\left(1-y_{j} t\right)\left(1-y_{j}^{-1} t\right)}{(1-t) \prod_{i=1}^{m}\left(1-x_{i} t\right)\left(1-x_{i}^{-1} t\right)}=\sum_{k=0}^{\infty} h_{k}\left(x_{1}, \ldots, x_{m}, y_{1}, \ldots, y_{n}\right) t^{k} .
$$

$O S P(2,2 n)$ : The supercharacter ring of $O S P(2,2 n)$ is generated by $h_{k}\left(x_{1}, y_{1}, \ldots, y_{n}\right), k \in \mathbb{Z}_{>0}$, where

$$
\chi_{G}(t)=\frac{\prod_{i=1}^{m}\left(1-y_{j} t\right)\left(1-y_{j}^{-1} t\right)}{\left(1-x_{1} t\right)\left(1-x_{1}^{-1} t\right)}=\sum_{k=0}^{\infty} h_{k}\left(x_{1}, y_{1}, \ldots, y_{n}\right) t^{k} .
$$

$O S P(2 m, 2 n), m \geq 2$ : The supercharacter ring of $O S P(2 m, 2 n)$ is generated by $\left.h_{k}\left(x_{1}, \ldots, x_{m}, y_{1}, \ldots, y_{n}\right)\right)$, $k \in \mathbb{Z}_{>0}$, where

$$
\chi_{G}(t)=\frac{\prod_{p=1}^{n}\left(1-y_{j} t\right)\left(1-y_{j}^{-1} t\right)}{\prod_{i=1}^{m}\left(1-x_{i} t\right)\left(1-x_{i}^{-1} t\right)}=\sum_{k=0}^{\infty} h_{k}\left(x_{1}, \ldots, x_{m}, y_{1}, \ldots, y_{n}\right) t^{k} .
$$

By Theorem 10, $d s_{x}\left(h_{k}^{\mathfrak{g}}\right)=\left.\left(h_{k}^{\mathfrak{g}}\right)\right|_{\beta=0}$. Since $\chi_{G}$ is $W$-invariant and $W \beta=\Delta_{\text {iso }}$ for any $\beta \in \Delta_{i s o}$, it suffices to consider the case that $\beta=\varepsilon_{1}-\delta_{1}$. In this case, $\beta=0$ if and only if $x_{1}=y_{1}$. It is not difficult to check that $\left.\chi_{G}(t)\right|_{x_{1}=y_{1}}=\chi_{G_{x}}$, and hence $d s_{x}\left(h_{k}^{\mathfrak{g}}\right)=h_{k}^{\mathfrak{g} x}$. Thus, all the generators of $\mathcal{J}_{G_{x}}$ are in the image of $d s_{x}$.

The general case for arbitrary $x \in X_{\mathfrak{g}}$ now follows from Corollary 11, since the composition of surjective maps is surjective.

Proposition 21. Let $\mathfrak{g}=\mathfrak{s l}(m \mid n), m \neq n$, or $\mathfrak{g}=\mathfrak{o s p}(m \mid 2 n)$. Then for any $x \in X_{\mathfrak{g}}$, the image of $d s_{x}: \mathcal{J}_{\mathfrak{g}} \rightarrow \mathcal{J}_{\mathfrak{g}_{x}}$ is the supercharacter ring $\mathcal{J}_{G_{x}}$ of the Lie supergroup $G_{x}$.

Proof. We use Theorem 20 and Theorem 10, together with the description of the rings $\mathcal{J}_{\mathfrak{g}}$ given by Sergeev and Veselov in [SV] to prove that the image of the map $d s_{x}: \mathcal{J}_{\mathfrak{g}} \rightarrow \mathcal{J}_{\mathfrak{g}_{x}}$ equals $\mathcal{J}_{G_{x}}$ in the case that $x \in \mathfrak{g}_{\beta}$ is an isotropic root $\beta$. The claim for any element $x \in X_{\mathfrak{g}}$ then follows Corollary 11.

The supercharacter ring of $\mathfrak{g}=\mathfrak{s l}(m \mid n), m \neq n$, is $\mathcal{J}_{\mathfrak{g}}=\mathcal{J}_{G} \oplus \bigoplus_{a \in \mathbb{C} / \mathbb{Z}} J(\mathfrak{g})_{a}$, where

$$
J(\mathfrak{g})_{a}=\left(x_{1} \cdots x_{n}\right)^{a} \Pi_{i, j}\left(1-x_{i} y_{j}^{-1}\right) \mathbb{Z}\left[x^{ \pm 1}, y^{ \pm 1}\right]_{0}^{S_{m} \times S_{n}},
$$

and $\mathbb{Z}\left[x^{ \pm 1}, y^{ \pm 1}\right]_{0}^{S_{m} \times S_{n}}$ is the quotient of the $\operatorname{ring} \mathbb{Z}\left[x_{1}^{ \pm 1}, \ldots, x_{m}^{ \pm 1}, y_{1}^{ \pm 1}, \ldots, y_{n}^{ \pm 1}\right]^{S_{m} \times S_{n}}$ by the ideal $\left\langle x_{1} \ldots x_{m}-y_{1} \ldots y_{n}\right\rangle$. Clearly, $\left.f\right|_{\beta=0}=\left.f\right|_{x_{i}=y_{j}}=0$ for any $f \in J(\mathfrak{g})_{a}$. Hence, $d s_{x}(f)=0$ for any $x \in X_{\mathfrak{g}}$ and $f \in J(\mathfrak{g})_{a}$. 
If $\mathfrak{g}=B(m \mid n), C(n+1)$ or $D(m \mid n)$, then $\mathcal{J}_{\mathfrak{g}}=\mathcal{J}_{G} \oplus \tilde{\mathcal{J}}$ and $d s_{x}(f)=0$ for all $f \in \tilde{\mathcal{J}}$. Indeed, for $\beta= \pm \varepsilon_{i} \pm \delta_{j}$ it is not difficult to check that $\left.f\right|_{\beta=0}=\left.f\right|_{x_{i}^{ \pm 1}=y_{j}^{ \pm 1}}=\left.f\right|_{u_{i}=v_{j}}=0$.

The supercharacter ring of $\mathfrak{g}=B(m \mid n)$ is $\mathcal{J}_{\mathfrak{g}}=\mathcal{J}_{G} \oplus J_{\mathfrak{g}, \frac{1}{2}}$, where

$$
J_{\mathfrak{g}, \frac{1}{2}}=\left\{\prod_{i=1}^{m}\left(x_{i}^{\frac{1}{2}}+x_{i}^{-\frac{1}{2}}\right) \prod_{i, j}\left(u_{i}-v_{j}\right) g \mid g \in \mathbb{Z}\left[u_{1}, \ldots, u_{m}, v_{1}, \ldots, v_{n}\right]^{S_{m} \times S_{n}}\right\} .
$$

The supercharacter ring of $\mathfrak{g}=C(n+1)$ is $\mathcal{J}_{\mathfrak{g}}=\mathcal{J}_{G} \oplus\left(J(\mathfrak{g})_{0}^{-} \oplus \bigoplus_{a \in \mathbb{C} / \mathbb{Z}} J(\mathfrak{g})_{a}\right)$, where

$$
\begin{gathered}
J(\mathfrak{g})_{0}^{-}=\left\{x_{1} \prod_{j=1}^{n}\left(u_{1}-v_{j}\right) g \mid g \in \mathbb{Z}\left[u_{1}, v_{1}, \ldots, v_{n}\right]^{S_{n}}\right\} \\
J(\mathfrak{g})_{a}=x_{1}^{a} \prod_{j=1}^{n}\left(1-x_{1} y_{j}\right)\left(1-x_{1} y_{j}^{-1}\right) \mathbb{Z}\left[x_{1}^{ \pm 1}, y_{1}^{ \pm 1}, \ldots, y_{n}^{ \pm 1}\right]^{W},
\end{gathered}
$$

The supercharacter ring of $\mathfrak{g}=D(m \mid n)$ is $\mathcal{J}_{\mathfrak{g}}=\mathcal{J}_{G} \oplus\left(J(\mathfrak{g})_{0}^{-} \oplus J_{\mathfrak{g}, \frac{1}{2}}\right)$, where

$$
\begin{gathered}
J(\mathfrak{g})_{0}^{-}=\left\{\omega \prod_{i, j}\left(u_{i}-v_{j}\right) g \mid g \in \mathbb{Z}\left[u_{1}, \ldots, u_{m}, v_{1}, \ldots, v_{n}\right]^{S_{m} \times S_{n}}\right\} \\
J_{\mathfrak{g}, \frac{1}{2}}=\prod_{i, j}\left(u_{i}-v_{j}\right)\left(\left(x_{1} \ldots x_{m}\right)^{\frac{1}{2}} \mathbb{Z}\left[u_{1}, \ldots, u_{m}, v_{1}, \ldots, v_{n}\right]\right)^{W} .
\end{gathered}
$$

Proposition 22. Let $\mathfrak{g}=\mathfrak{g l}(m \mid n)$ and $x \in X_{\mathfrak{g}}$. The image of $d s_{x}: \mathcal{J}_{\mathfrak{g}} \rightarrow \mathcal{J}_{\mathfrak{g}_{x}}$ is

$$
\bigoplus_{a \in \mathbb{C} / \mathbb{Z}}\left(x_{1} \cdots x_{m-k}\right)^{a}\left(y_{1} \cdots y_{n-k}\right)^{-a} \mathcal{J}_{G_{x}}
$$

where $k$ is the size of $\psi(x) \in S_{\mathfrak{g}}$ under the bijection $\psi: X_{\mathfrak{g}} / G_{\overline{0}} \rightarrow S_{\mathfrak{g}} / W$, and $\mathcal{J}_{G_{x}}$ is the supercharacter ring of the Lie supergroup $G_{x}$.

Proof. By Sergeev and Veselov in [SV], the supercharacter ring of $\mathfrak{g l}(m \mid n)$ is $\mathcal{J}_{\mathfrak{g}}=\bigoplus_{a, b \in \mathbb{C} / \mathbb{Z}} J(\mathfrak{g})_{a, b}$ where $J(\mathfrak{g})_{0,0}=\mathcal{J}_{G}$,

$$
J(\mathfrak{g})_{a, b}=\left(x_{1} \cdots x_{m}\right)^{a}\left(y_{1} \cdots y_{n}\right)^{-a} J(\mathfrak{g})_{0,0}
$$

when $a+b \in \mathbb{Z}$, but $a \notin \mathbb{Z}$, and

$$
J(\mathfrak{g})_{a, b}=\left(x_{1} \cdots x_{m}\right)^{a}\left(y_{1} \cdots y_{n}\right)^{b} \Pi_{i, j}\left(1-x_{i} y_{j}^{-1}\right) \mathbb{Z}\left[x_{1}^{ \pm 1}, \ldots, x_{m}^{ \pm 1}, y_{1}^{ \pm 1}, \ldots, y_{n}^{ \pm 1}\right]^{S_{m} \times S_{n}}
$$

when $a+b \notin \mathbb{Z}$.

Then we have that $\left.f\right|_{x_{i}=y_{j}}=0$ for any $f \in J(\mathfrak{g})_{a, b}$ with $a+b \notin \mathbb{Z}$. By Theorem 20, $d s_{x}\left(J(\mathfrak{g})_{0,0}\right)=J\left(\mathfrak{g}_{x}\right)_{0,0}$. Since $d s_{x}(f)=\left.f\right|_{x_{r_{i}}=y_{s_{i}} ; i=1, \ldots, k}$ by Theorem 10, we have that

$$
d s_{x}\left(J(\mathfrak{g})_{a, b}\right)=\left(x_{1} \cdots x_{m-k}\right)^{a}\left(y_{1} \cdots y_{n-k}\right)^{-a} J\left(\mathfrak{g}_{x}\right)_{0,0}=J\left(\mathfrak{g}_{x}\right)_{a, b}
$$

when $a+b \in \mathbb{Z}$, but $a \notin \mathbb{Z}$. 
5.2. The image of $d s_{x}$ for the exceptional Lie superalgebras. In this section, we describe the image of $d s_{x}$ for the Lie superalgebras $G(3), F(4)$ and $D(2,1, \alpha)$, using the explicit description of the supercharacter rings given by Sergeev and Veselov in [SV, Section 7].

Since $G(3), F(4)$ and $D(2,1, \alpha)$ have defect 1 , we may assume that $x \in \mathfrak{g}_{\beta}$ for some isotropic root $\beta$. Moreover, since $W \beta=\Delta_{\overline{1}}$, it suffices to describe the image for a fixed choice of $\beta$.

5.2.1. $G(3)$. Let $\beta=\varepsilon_{3}+\delta_{1}$. Then $\mathfrak{g}_{x} \cong \mathfrak{s l}(2)$ with $\Delta_{x}=\left\{ \pm\left(\varepsilon_{1}-\varepsilon_{2}\right)\right\}$. The supercharacter ring of $G(3)$ is

$$
\mathcal{J}_{\mathfrak{g}}=\left\{g(w)+\left(v_{1}-u_{1}\right)\left(v_{1}-u_{2}\right)\left(v_{1}-u_{3}\right) h \mid h \in \mathbb{Z}\left[u_{1}, u_{2}, u_{3}, v_{1}\right]^{S_{3}}, g \in \mathbb{Z}[w]\right\},
$$

where $y_{1}=e^{\delta_{1}}, v_{1}=y_{1}+y_{1}^{-1}, x_{i}=e^{\varepsilon_{i}}, u_{i}=x_{i}+x_{i}^{-1}$ for $i=1,2,3$ and

$$
w=v_{1}^{2}-v_{1}\left(u_{1}+u_{2}+u_{3}+1\right)+u_{1} u_{2}+u_{1} u_{3}+u_{2} u_{3} \text {. }
$$

Note that $x_{1} x_{2} x_{3}=1$, so $u_{3}=x_{1} x_{2}+x_{1}^{-1} x_{2}^{-1}$.

Theorem 10 implies that $d s_{x}(f)=\left.f\right|_{y_{1}=x_{3}^{-1}=x_{1} x_{2}}$ for every $f \in \mathcal{J}_{\mathfrak{g}}$. Hence, $d s_{x}(f)=d s_{x}(g(w))$ since $\left.\left(v_{1}-u_{3}\right)\right|_{y_{1}=x_{3}^{-1}=x_{1} x_{2}}=0$. Thus, the image of $d s_{x}$ is the polynomial ring $\mathbb{Z}\left[w_{x}\right]$ generated by the element

$$
w_{x}:=\left.w\right|_{y_{1}=x_{3}^{-1}=x_{1} x_{2}}=\frac{x_{1}}{x_{2}}+\frac{x_{2}}{x_{1}} \in \mathcal{J}_{\mathfrak{g}_{x}} .
$$

Note that $w_{x}+1$ is the supercharacter of the adjoint representation of $\mathfrak{s l}(2)$, and that $\frac{x_{1}}{x_{2}}+\frac{x_{2}}{x_{1}}$ equals $x_{1}^{2}+x_{2}^{2}$ in $\mathcal{J}_{\mathfrak{g}_{x}}$ due to the relation $x_{1} x_{2}=1$. Finally, we obtain that

$$
\operatorname{Im} d s_{x}=\mathbb{Z}\left[x_{1}^{2}+x_{2}^{-2}\right] \subsetneq \mathcal{J}_{G_{x}}=\mathcal{J}_{S L(2)}=\mathbb{Z}\left[x_{1}^{ \pm 1}, x_{2}^{ \pm 1}\right]^{S_{2}} /\left\langle x_{1} x_{2}-1\right\rangle \cong \mathbb{Z}\left[x_{1}+x_{1}^{-1}\right] .
$$

5.2.2. $F(4)$. Let $\beta=\frac{1}{2}\left(\varepsilon_{1}+\varepsilon_{2}+\varepsilon_{3}-\delta_{1}\right)$. Then $\mathfrak{g}_{x} \cong \mathfrak{s l}(3)$ with $\Delta_{x}=\left\{\varepsilon_{i}-\varepsilon_{j} \mid 1 \leq i, j \leq 3\right\}$. The supercharacter ring of $F(4)$ is

$$
\mathcal{J}_{\mathfrak{g}}=\left\{g\left(w_{1}, w_{2}\right)+Q h \mid h \in \mathbb{Z}\left[x_{1}^{ \pm 2}, x_{2}^{ \pm 2}, x_{3}^{ \pm 2},\left(x_{1} x_{2} x_{3}\right)^{ \pm 1}, y_{1}^{ \pm 1}\right]^{W_{0}}, g \in \mathbb{Z}\left[w_{1}, w_{2}\right]\right\}
$$

where $y_{1}=e^{\frac{1}{2} \delta_{1}}, x_{i}=e^{\frac{1}{2} \varepsilon_{i}}$ for $i=1,2,3$, and

$$
\begin{aligned}
Q & =\left(y_{1}+y_{1}^{-1}-x_{1} x_{2} x_{3}-x_{1}^{-1} x_{2}^{-1} x_{3}^{-1}\right) \prod_{i=1}^{3}\left(y_{1}+y_{1}^{-1}-\frac{x_{1} x_{2} x_{3}}{x_{i}^{2}}-\frac{x_{i}^{2}}{x_{1} x_{2} x_{3}}\right) \\
w_{k} & =\sum_{i \neq j} \frac{x_{i}^{2 k}}{x_{j}^{2 k}}+\sum_{i=1}^{3}\left(x_{i}^{2 k}+x_{i}^{-2 k}\right)+y_{1}^{2 k}+y_{1}^{-2 k}-\left(y_{1}^{k}+y_{1}^{-k}\right) \prod_{i=1}^{3}\left(x_{i}^{k}+x_{i}^{-k}\right), \quad k=1,2 .
\end{aligned}
$$

Theorem 10 implies that $d s_{x}(f)=\left.f\right|_{x_{1} x_{2} x_{3}=y_{1}}$ for every $f \in \mathcal{J}_{\mathfrak{g}}$. Hence, $d s_{x}(f)=d s_{x}\left(g\left(w_{1}, w_{2}\right)\right)$ since $\left.Q\right|_{x_{1} x_{2} x_{3}=y_{1}}=0$. Thus, the image of $d s_{x}$ is generated by the elements

$$
\begin{aligned}
& w_{x}^{1}:=\left.w_{1}\right|_{x_{1} x_{2} x_{3}=y_{1}}=\sum_{i \neq j} \frac{x_{i}^{2}}{x_{j}^{2}} \\
& w_{x}^{2}:=\left.w_{2}\right|_{x_{1} x_{2} x_{3}=y_{1}}=\sum_{i \neq j} \frac{x_{i}^{4}}{x_{j}^{4}},
\end{aligned}
$$

and is a proper subring of $\mathcal{J}_{G_{x}}=\mathcal{J}_{S L(3)}=\mathbb{Z}\left[x_{1}^{ \pm 1}, x_{2}^{ \pm 1}, x_{3}^{ \pm}\right]^{S_{3}} /\left\langle x_{1} x_{2} x_{3}-1\right\rangle$. 
5.2.3. $D(2,1, \alpha)$. Let $\beta=\varepsilon_{1}-\varepsilon_{2}-\varepsilon_{3}$. Then $\mathfrak{g}_{x} \cong \mathbb{C}$.

If $\alpha \notin \mathbb{Q}$, then the supercharacter ring of $D(2,1, \alpha)$ is

$$
\mathcal{J}_{\mathfrak{g}}=\left\{c+Q h \mid c \in \mathbb{Z}, h \in \mathbb{Z}\left[u_{1}, u_{2}, u_{3}\right]\right\},
$$

where $x_{i}:=e^{\varepsilon_{i}}, u_{i}=x_{i}+x_{i}^{-1}$ for $i=1,2,3$ and

$$
\begin{aligned}
Q & =\left(x_{1}-x_{2} x_{3}\right)\left(x_{2}-x_{1} x_{3}\right)\left(x_{3}-x_{1} x_{2}\right)\left(1-x_{1} x_{2} x_{3}\right) x_{1}^{-2} x_{2}^{-2} x_{3}^{-2} \\
& =u_{1}^{2}+u_{2}^{2}+u_{3}^{2}-u_{1} u_{2} u_{3}-4 .
\end{aligned}
$$

If $\alpha=\frac{p}{q} \in \mathbb{Q}$, then the supercharacter ring of $D(2,1, \alpha)$ is

$$
\mathcal{J}_{\mathfrak{g}}=\left\{g\left(w_{\alpha}\right)+Q h \mid g \in \mathbb{Z}\left[w_{\alpha}\right], h \in \mathbb{Z}\left[u_{1}, u_{2}, u_{3}\right]\right\},
$$

where

$$
w_{\alpha}=\left(x_{1}+x_{1}^{-1}-x_{2} x_{3}-x_{2}^{-1} x_{3}^{-1}\right) \frac{\left(x_{2}^{p}-x_{2}^{-p}\right)\left(x_{3}^{q}-x_{3}^{-q}\right)}{\left(x_{2}-x_{2}^{-1}\right)\left(x_{3}-x_{3}^{-1}\right)}+x_{2}^{p} x_{3}^{-q}+x_{2}^{-p} x_{3}^{q} .
$$

By Theorem [10, $d s_{x}(f)=\left.f\right|_{x_{1}=x_{2} x_{3}}$ for every $f \in \mathcal{J}_{\mathfrak{g}}$. Since $\left.Q\right|_{x_{1}=x_{2} x_{3}}=0, d s_{x}(f)=c$ for some $c \in \mathbb{Z}$ when $\alpha \notin \mathbb{Q}$, while $d s_{x}(f)=d s_{x}\left(g\left(w_{\alpha}\right)\right)$ when $\alpha \in \mathbb{Q}$. Thus the image of $d s_{x}$ is $\mathbb{Z} \subset \mathcal{J}_{\mathbb{C}}$ when $\alpha \notin \mathbb{Q}$ and the image is $\mathbb{Z}\left[w_{\alpha}\right] \subset \mathcal{J}_{\mathbb{C}}$ when $\alpha \in \mathbb{Q}$.

\section{REFERENCES}

[BKN] B. Boe, J. Kujawa, D. Nakano, Complexity for modules over the Lie superalgebra gl $(\mathrm{m} / \mathrm{n})$, Compositio Math. (2012), doi:10.1112/S0010437X12000231.

[BK] J. Brundan and J. Kujawa, A new proof of the Mullineux conjecture, J. Algebraic Combin. 18 (2003), 13-39. MR 2002217. Zbl 1043.20006. doi: 10.1023/A: 1025113308552.

[CH] J. Comes, T. Heidersdorf, Thick Ideals in Deligne's category Rep $\left(O_{\delta}\right)$, J. Algebra 480 (2017), $237-265$.

[CW] S.J. Cheng, W. Wang, Dualities and Representations of Lie Superalgebras, Graduate Studies in Mathematics 144, AMS, 2012.

[DS] M. Duflo, V. Serganova, On associated variety for Lie superalgebras, arXiv:math/0507198 (2005).

[EHS] I. Entova Aizenbud, V. Serganova, V. Hinich, Deligne categories and the limit of categories Rep $(G L(m \mid n))$, arXiv:1511.07699 (2015).

[GK] M. Gorelik, V.G. Kac, Characters of (relatively) integrable modules over affine Lie superalgebras, Japan. J. Math (2015), Volume 10, Issue 2, 135--235.

[GS] C. Gruson, V. Serganova, Cohomology of generalized supergrassmannians and character formulae for basic classical Lie superalgebras, Proc. Lond. Math. Soc. 101 (2010) 852--892.

[H] T. Heidersdorf, On supergroups and their semisimplified representation categories, arXiv:1512.03420 (2015).

[HW1] T. Heidersdorf, R. Weissauer, Cohomological Tensor Functors on Representations of the General Linear Supergroup, arXiv:1406.0321 (2014).

[HW2] T. Heidersdorf, R. Weissauer, Pieri type rules and Gl(2|2) tensor products, arXiv:1509.07443 (2015).

[KW1] V.G. Kac, M. Wakimoto, Integrable highest weight modules for affine Lie superalgebras and number theory, in Lie Theory and Geometry, 415--456, Progress in Math., \{123\}, Birkhauser Boston, MA, 1994.

[KW2] V.G. Kac, M. Wakimoto, Representations of affine superalgebras and Mock theta functions, Trans. Groups, Vol. 19, No. 2 2014, 383-455.

[Ma] I.G. Macdonald, Symmetric Functions and Hall Polynomials, second ed., Oxford Math. Monogr., Oxford Univ. Press, New York, 1995. MR 1354144. Zbl 0824.05059.

[Mu] I.M. Musson, Lie superalgebras and enveloping algebras, Graduate Studies in Mathematics, vol. 131, 2012.

[SV] A.N. Sergeev, A.P. Veselov, Grothendieck rings of basic classical Lie superalgebras, Ann. Math, 173 (2011), $663-703$.

[S11] V. Serganova, On the superdimension of an irreducible representation of a basic classical Lie superalgebra, Supersymmetry in Mathematics and Physics, Lecture Notes in Mathematics (2011), 253--273.

[S14] V. Serganova, Finite dimensional representations of algebraic supergroups, Proceedings of the International Congress of Mathematicians (Seoul), 2014.

C.H.: Dept. of Mathematics, Weizmann Institute \& ORT Braude College, crystal.hoyt@weizmann.ac.il

S.R.: Dept. of Mathematics, Bar-Ilan University, shifra.reif@biu.ac.il 